\title{
Post-nonclassical science, modern design and the idea of sociocultural interactions' synchronicity
}

\author{
Marina Plotnikova ${ }^{1}$, Elena Polozhenkova ${ }^{1}$, Daria Burmenskaya ${ }^{1,{ }^{*}}$ \\ ${ }^{1}$ Don State Technical University, 1, Gagarin sq., 344000, Rostov-on-Don, Russia
}

\begin{abstract}
This article is devoted to the science place problem study in culture, its relationship with other sociocultural phenomena. The purpose of the article is to identify the post-non-classical science relationship specifics with such a phenomenon of modern culture as design. The identification of this specificity can serve as an argument that testifies to the rootedness of post-non-classical science in modern culture. To achieve this goal, the authors set the following tasks: 1) analysis of the science sociocultural determination levels and types; 2) the disclosure of the postnon-classical science and modern design features; 3) the identification of the correlation between them. The authors proceed from the idea of synchronism or parallelism as a form of interaction of sociocultural phenomena and processes. A comparative analysis of the post-nonclassical science and modern design main characteristics reveals their common features. The authors associate the existence of these correlations with the sociocultural, worldview influence of postmodernism. The obtained result confirms the idea of the post-non-classical science rootedness in modern culture.
\end{abstract}

\section{Introduction}

The relevance of addressing the problem of the science place in culture, its relationships with other sociocultural phenomena is associated with a certain specificity of modern scientific knowledge. First of all, it should be noted that under the influence of the specialization extremely increased in the XX-XXI century, on the one hand, the science abstractness and mathematization, on the other hand, an illusion about its autonomy with respect to culture, various forms of spiritual activities such as philosophy, art, religion. It is regarded as a kind of "ivory tower", a completely sovereign, intellectual enterprise, and the understanding of its original semantic purpose is lost. In addition, the legitimacy of the scientists' social responsibility question for the consequences of certain scientific research and discoveries becomes very doubtful.

In its turn, the scientist version of the science phenomenon is sharply criticized due to the presence of those harmful environmental and moral-anthropological consequences that are associated with modern scientific and technological progress. The responsibility for

\footnotetext{
* Corresponding author: darya-burmenskay@mail.ru
} 
almost all the troubles of modern civilization and culture lies in modern science. Such criticism, brought to anti-scientism, in its radicalism threatens with the scientific rationality destruction as such, that is, the destruction of an important part of the European civilization and culture foundation. The presence of such opposing positions in the interpretation of the relationship between science and culture determines the relevance of further research on this issue.

The purpose of this article is to identify the specifics of the post-non-classical science relationship with other phenomena of modern culture, namely with design. The identification of this specificity can serve as an argument that testifies to the rootedness of post-non-classical science in modern culture. To achieve this goal, the following tasks are expected to be solved:

1) analysis of the levels and types of the science sociocultural determination;

2) the disclosure of the post-non-classical science and modern design features;

3) identification of the correlations between them.

\section{Materials and methods}

To achieve this goal, the authors use a number of methods, methodological procedures and operations. To explain the parallelism of post-non-classical science and modern design, the authors use the deductive-nomological type of explanation, which was once proposed by K. Hempel, (later called the Hempel - Oppenheim scheme). In this explanatory model, as known, the scheme of explanation is used through the general law, that is, the explanation in its logical structure is a reasoning of the deductive type from general to particular. Identifying the correlation between the post-non-classical science and modern design, the authors use the methodological procedure of comparative analysis, as well as general logical operations of analysis, synthesis, deduction, induction, generalization, abstraction, dialectical principles of historicism and concreteness of consideration.

\section{Discussions and Results}

In this paper, we do not set the task of considering the problem of the sociocultural determination of science in all its breadth. Similar studies were carried out by us in a number of previous works $[1,2]$. In them, we rely, as already noted, on a fairly wellfounded concept of sociocultural determination of science by E.A. Mamchur. She distinguishes 3 levels and types of socio-cultural influence on the scientific knowledge: the sociocultural nature of cognition, sociocultural conditioning, sociocultural determination [3]. In this case, the researcher recognizes the legitimacy of the first two levels of the sociocultural factors' influence on the dynamics of science, rejecting the third of them. This position is justified by the fact that the assumption of the thesis: "sociocultural determination in the limit leads to the conclusion that the content of scientific knowledge ... represents some kind of social construction As a result, the preservation of epistemological autonomy by science ... is very doubtful" [2].

However, it seems that the principle of determination should be interpreted more broadly. It is unjustified to reduce it only to causality, which is a connection that has a genetic, generative nature, as well as the precedence of one phenomenon in time to another [3]. In addition to the causal determination, there are also the non-causal relationships (conditional, functional, systemic, governing, correlative, "states' relationship" and others closely related to causality, but not reducible to it).

E.A. Mamchur draws particular attention to the special importance in the relationship between the sociocultural context and the scientific knowledge of synchronous 
determination, to which the states' relationship and the correlative connections relates [3]. The existence of this type of relationship is one of the first to be revealed by C. Jung, distinguishing three types of the relations between the phenomena:

1) causal;

2) acausal (random);

3) synchronous, which are acausal (non-causal), but not the random coincidences, but meaningful, meaningful connections.

Studying the human psyche phenomena, K. Jung defines the synchronism as "a stable, repetitive appearance of a certain mental state of an individual, simultaneous with some external event, which is recognized as a meaningful parallel" [4].

If we turn to the history of culture, we can find some similar parallelism between various sociocultural phenomena in a given era. For example, it is no coincidence that the 17th century in the history of European culture is considered the century of Reason. Rationalism in philosophy, science and even religion is interfaced with classicism in art, which embodies rationalism in an artistic form. In ancient Greek culture, the Aristotelian concept of "place" and the "round sculpture" of antiquity reflect the peculiar perception of space by ancient man. At the same time, the infinite homogeneous space of the physics of Galileo and Newton correlates with a direct linear perspective in modern painting. Similar examples can be continued.

We ask ourselves: in such cultural parallels, are some phenomena the cause or foundation of the others? Apparently not, rather they arise quite synchronously and affect each other without entering into a relationship of generation. Their common source is the features of a particular historical era people's life, worldview, mentality, lifestyle. It turns out that the totality of the diverse relationships and the correlations that permeate the scientific knowledge goes into the general worldview of a particular era.

In this regard, returning to the question of the "socio-cultural determination" concept legitimacy it should be noted that if "socio-cultural determination" is understood to be the causeless synchronistic connections, then this concept is quite acceptable to use as a synonymous to the "socio-cultural conditioning" concept.

Further, if we turn to post-non-classical science and its foundations, will we find a similar parallelism between it and other sociocultural phenomena?

It is generally recognized that the conceptual and methodological core of post-nonclassical science is a synthesis of the systems' self-organization synergetic theory and the principle of global evolutionism, the study of which is devoted to an extensive array of the scientific works by I. R. Prigogine, G. Haken, E. Agazzi, N. I. Moiseeva, K.Kh. Delokarova, R.S. Karpinskaya, A.P. Ogurtsova and other scientists. Without going into a detailed analysis of these theoretical and methodological formations' specifics, we dwell on those philosophical and worldview foundations that are largely due to them.

So, in modern science a new picture of the world is forming, in which the universe appears as a complex nonequilibrium system of interactions of micro - macro - and megaspheres. The instability of the phenomena of this system gives rise to an awareness of the limitations of human capabilities in their control. A different interpretation, in comparison with classical science, receives the principle of determinism. In immanently self-organizing systems, not a rigid causal connection, but acausal, random dominates. Moreover, randomness plays a decisive role in the bifurcation period, which determines non-linearity, that is, multivariance, alternative to the evolutionary development of the system. After "choosing" a certain development path at a bifurcation point, determinism again dominates in the system, etc. In other words, in a nonequilibrium system, the determination mechanism is an alternation of causal and acausal connections.

Along with the formation of a new world picture, the norms and ideals of science are being transformed. The value aspect becomes their integral component. 
Thus, along with its truthfulness, an important criterion of the scientific knowledge is the consistency criterion with universal values and ideals, the attitude to the survival of mankind in the modern world, as well as the rational and effective structure of human life. At the same time, a theoretical and methodological setting is being formed, suggesting the need to take into account the presence of a genetic connection between inorganic and organic nature, as well as a new understanding of the epistemological subject and man in general as part of nature. Awareness of the nature human world proportionality leads to a critical rethinking of the human domination idea over it, so rooted in classical new European natural science.

A characteristic feature of post-non-classical science is its dialogic nature. She recognizes the diversity of approaches, concepts, communication of individuals and representatives of different cultures. Many researchers come to the conclusion that dialogue, involving a variety of visions of a particular problem and the possibility of effective communication between their carriers, is becoming a kind of new form of rationality, characteristic not only of the humanities, but also of the natural sciences. In general, the plurality and diversity idea plays a special role not only in modern epistemology, but also in scientific ontology.

In this regard, E.A. Mamchur draws attention to the fact that even in physics, completely indifferent to any mindset, "the idea of diversity is now becoming more popular than the idea of uniformity" [4]. The researcher compares in this aspect classical physics with non-classical science, and discovers a fundamental difference between them. If in classical natural science the idea of symmetry is indisputably given priority, then in modern physical cognition the idea of breaking symmetry is considered more fruitful, since breaking symmetry is considered as the source of the particles' variety reigning in the world. In this regard, E.A. Mamchur notes that from the point of view of many serious researchers, "the ontology of modern physics" is not the unity sought for and always found in classical physics, but a multitude of hierarchically ordered, but not reducible, models [4].

A somewhat different emphasis is placed by those researchers who, for the real pluralism of disciplinary ontologies, see the possibility of creating a unified scientific picture of the world that integrates knowledge of inorganic and organic nature, as well as socio-cultural life. In this regard, T.P. Matyash emphasizes that the basis of the modern scientific picture of the world is the "unity in the diversity of various disciplinary ontologies" [5]. At the same time, the scientific picture of the world is adapting to the modern cultural worldview universals, which we mentioned above, and at the same time changing them.

Next, we turn to the modern design phenomenon, one of the most striking and characteristic manifestations of modern culture, which also undergoes significant changes in the second half of the 20th century. It should be noted that regarding the time and cultural-historical conditions of the design emergence, we adhere to a point of the view which supporters (T.Yu. Bystrova, L.A. Zelenov, V.O. Pigulevsky, V.F. Runge, V.A. Schurov etc.) connect its origin with the industrial era, namely, the years of the great American depression in the thirties of the 20th century.

At this time, American industry was experiencing difficulties in marketing manufactured goods, and, accordingly, initially the design was used in the mass production of the new household items and technical products. However, by the middle of the last century, its claims to globalization and universality were realized, since the designer became an integral participant in the design of almost all products' manufacturing - from equipment to clothing.

The ideological design basis of this period is the system of values and ideals of modernity as one of the main streams of a new European culture. In this regard, the modernist idea of rational reorganization of all reality is transformed in the theory and 
practice of design. It is embodied in the ideal of "a unified style-formation of the entire subject environment of a person, from housing architecture to the specifics of individual household things". As a result, "a certain integrity possessing mutual flow and indissolubility of aesthetic and functional" is cultivated in design thinking [6]. Functionalist theory affirms the design ability in alliance with technology to improve this world and solve all possible social problems.

However, the destruction of the positivistic picture of the world and modernist illusions by the sixties of the last century clearly indicates the end of the modernist era. The sociocultural situation of postmodernism is being formed. Life shows that neither rationalism nor functionalism can explain the changes taking place in society. In Western Europe, radical anti-functionalist theories are emerging that sharply criticize the slogan: "Form follows function": a thing should not fulfill only a technical, utilitarian function.

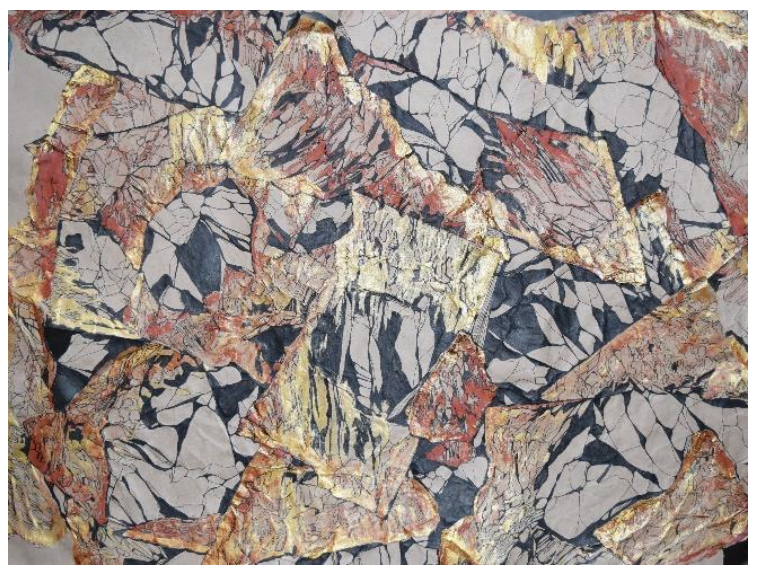

Fig. 1. Author's graphics Plotnikova M. G. "Textured beginning".

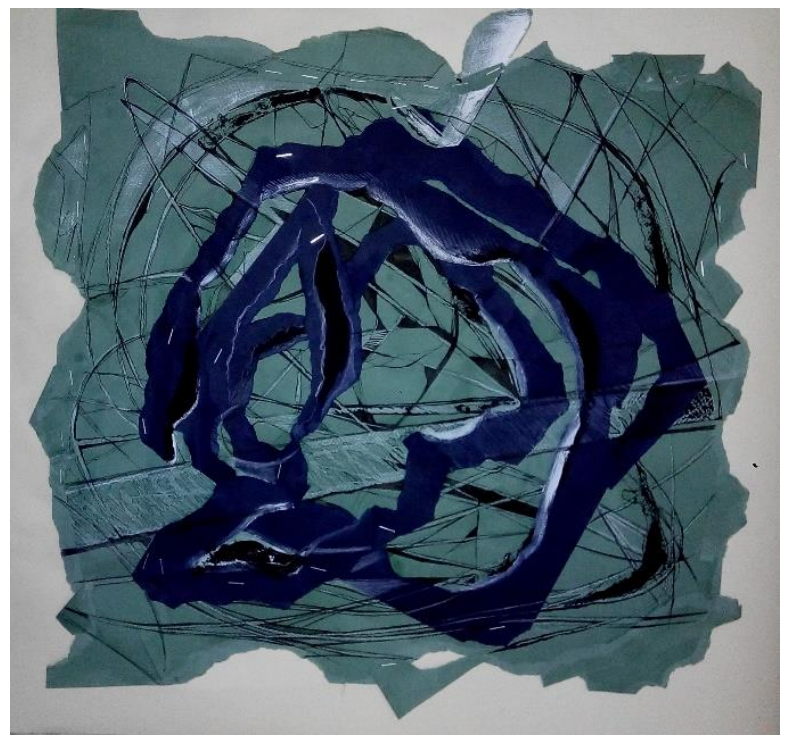

Fig. 2. Author's graphics Plotnikova M. G. "the Name is for sale".

In the postmodern situation in the field of design, there is a "wide variety of types of beauty and benefit correlation - from functionalism to styling, and the functional redundancy of the real world determines the variability of a person's attitude to things - 
alienation, consumption, personification, desacralization, glamorization, fetishization, etc. Use in design brand strategies, quoting styles and the impressionsэ formation indicate a rejection of modernist attitudes in favor of postmodernism: "form absorbs function" (F. Bayer)" [6].

However, postmodern shaping is very specific. Based on the idea of denying any novelty or primordiality in the ontological aspect, postmodernism borrows art from the existing art systems, both past and more modern styles are cited. At the same time, artists working in a postmodern manner are sometimes quite ironic, trying to demonstrate that there is nothing worthy the attention for the apparent seriousness of an artistic or intellectual phenomenon. In this regard, V.O. Pigulevsky, comparing the peculiarities of modern and postmodern cultures, emphasizes their significant difference, namely that the design culture of modernity is characterized by the dominance of a transcendental subject producing “... total design, the utopian world-order project designed to solve the social problems by means of design. The turn to objectivity, physicality and textuality (in postmodernism - M. Plotnikova) relies on the spontaneous organization of the living environment, but, being carried away by quoting the past, loses its perspectives, vision of the future" [7].

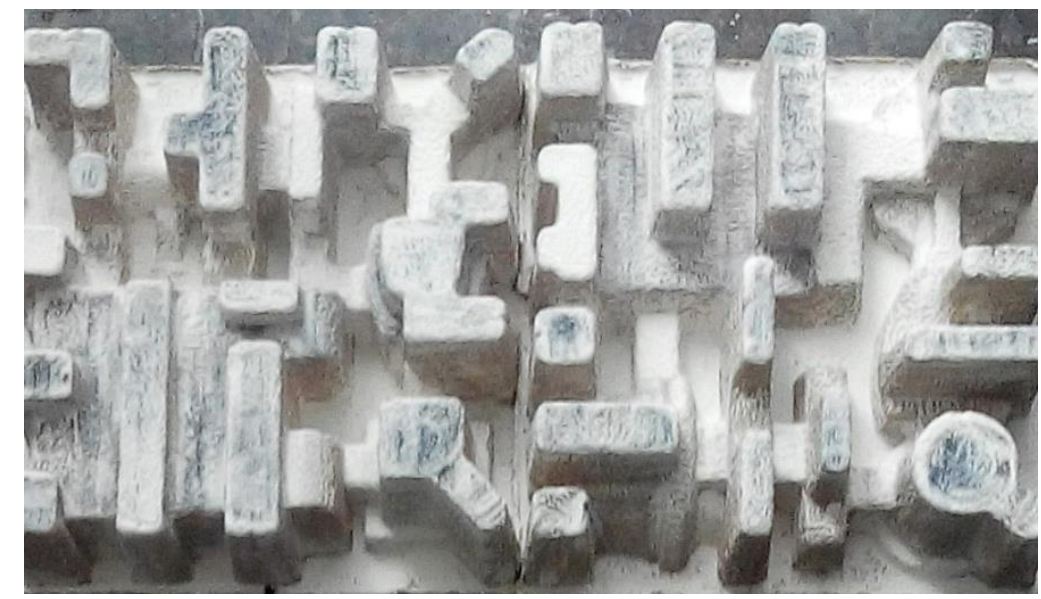

Fig. 3. Author's panel of Plotnikova M.G. "Apotheosis of the future».

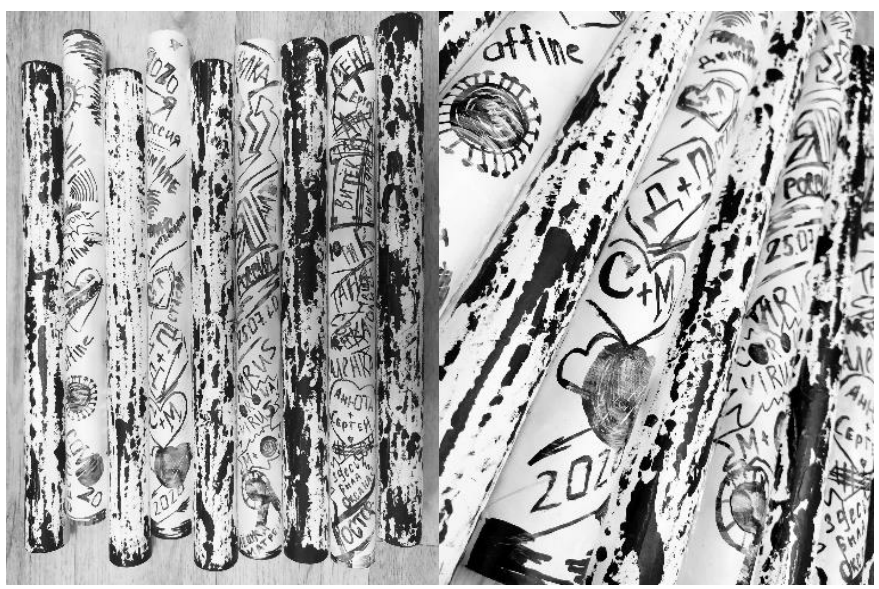

Fig. 4. Author's work of Plotnikova M. G. art object «Tears of birch». 
As noted above, the design is extremely symbolic. Brand names practically replace the things themselves, gain an independent life. This feature is especially brightly represented in the world of fashion. Here the inscriptions and labels are inseparable from clothes and shoes, accessories.

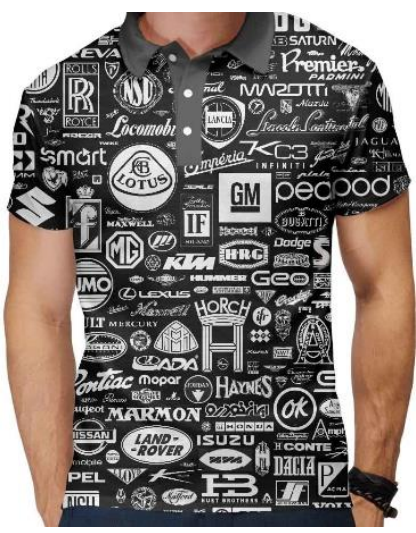

Fig. 5. Brand names in clothing.

In addition to the symbolism of the design product, communicativeness becomes its essential characteristic in the postmodern situation. It acts as a peculiar way of communication. Since the new computer technologies arise as a result of modern computerization and informatization, the products of a person's material and spiritual activities acquire a new form of existence, are fixed in the iconic sociocultural systems. As a result, a cultural space is formed that draws the individual into a diverse dialogue with the outside world.

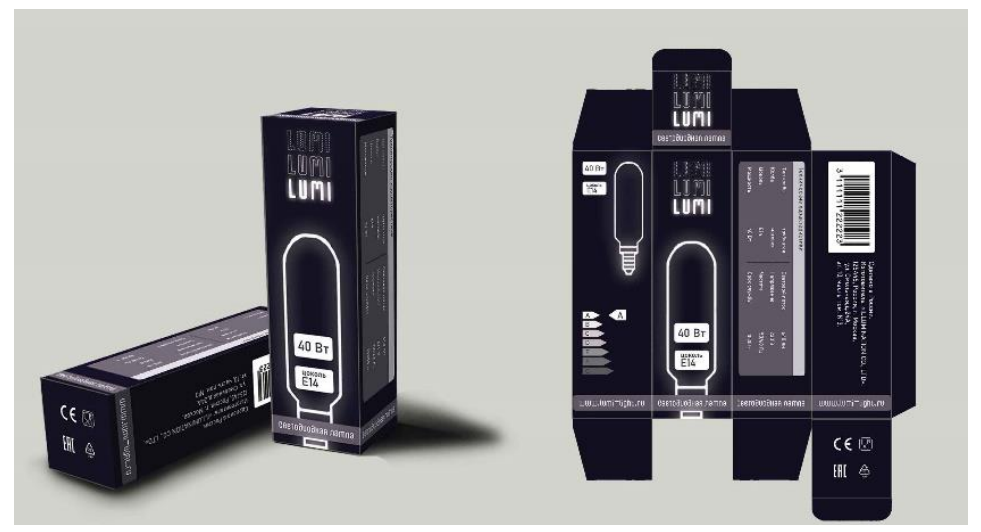

Fig. 6. Author's work of the student of the Department "Design and construction of light industry products" of DSTU Anastasia Baimova «Design solution of packaging graphics «LUM».

Moreover, the relationship of design with communication systems is a complex multilateral interaction, that is, it, in turn, experiences the influence of various social and cultural contexts. Therefore, as noted by J. Habermas, "a turn toward postmodernism means a change in the production paradigm to the theory of communicative action, an understanding of practice as the "everyday world" [8]. In this world, things are transformed into signs, some communication tools through which a person interacts with other people. In postmodern literature, this idea was developed in particular in the works of $\mathrm{J}$. Baudrillard. The focus of the French philosopher's attention, exploring a thing as a 
communication system, is the special role of the image, which he plays in the consumption processes [9].

So, as a study result of the main characteristics of post-non-classical science and such a sociocultural phenomenon as modern design, the following results were obtained.

The first one. The scientific picture of the world, the norms and ideals of post-nonclassical science, are characterized by a number of specific properties that distinguish it from the previous historical conditions, especially from the foundations of classical new European natural science. The idea of pluralism, diversity penetrates deeply into scientific ontology and epistemology. Pluralism in comprehending the modern picture of the world and the methodological arsenal of its knowledge determines the communicativeness, modern science dialogic nature.

The second one. Modern design, as a result of transformation in the cultural and historical situation of postmodernism, acquires certain specific features, among which, first of all, the idea of variability, the beauty and benefit correlation types' diversity, pluralism in relation to a person to a thing, style diversity is highlighted. This fundamental feature is closely related to the communicative nature of the sociocultural phenomenon, which acts as a kind of communication method.

The third one. The presence of similar characteristics of pluralism, diversity, communicativeness, identified in post-non-classical science and modern design, is explained, in our opinion, by the synchronous determination, parallelism of various sociocultural phenomena developing in a certain historical period. The source of parallelism is the peculiarities of lifestyle, thoughts, worldview, experiences of a modern man in the postmodern culture conditions.

\section{Summary}

A comparative analysis of the post-non-classical science foundations and the specifics of modern design showed that they have common specific features, such as pluralism and communication. The presence of the similar features' data is not an evidence of a causal relationship between them. It defines the parallelism, temporal and semantic synchronicity of science and modern design, therefore, of the rootedness of science in culture, its connections and interactions with other sociocultural phenomena in forms of causal determination.

\section{References}

1. D.N. Burmese, Religious Origins of New European Science (The dissertation for the degree of candidate of philosophical sciences, Rostov-on-Don, 2012)

2. D.N. Burmenskaya, E.Yu. Polozhenkova, Genesis of New European Science (Theological Context, Shahtyi, 2014)

3. E.M. Mamchur, Problems of the sociocultural determination of scientific knowledge To discussions in modern post-positivistic philosophy of science (Science, Moscow, 1987)

4. R.Z. Smith, Introduction to the Electronic Age (E-Publishing Inc., New York, 1999)

5. E.A. Mamchur, Images of science in modern culture (Canon, Moscow, 2008)

6. T.P. Matyash, Antique and Scientific Rationality: Historical Transformations, Humanitarian Diary (Rost.gos Publishing House. University, Rostov-on-Don, 2004) 
7. M.G. Plotnikova, Design of a thing: cultural-historical transformations (Abstract of dissertation for the degree of candidate of philosophical sciences, Rostov-on-Don, 2014)

8. V.O. Pigulevsky, Humanities and Social Sciences 3, 62-69 (2012) http://hsesonline.ru/2012_03.html

9. Y. Habermas, Philosophical Discourse on Art Nouveau (Moscow, 2008)

10. J. Baudrillard, System of Things (Rudomino, Moscow, 2001) 